\title{
Belgeo
}

Revue belge de géographie

$1 \mid 2013$

Modelling and benchmarking of borders

\section{Guest editorial : Modèles de frontières}

Grégory Hamez, Anne-Laure Amilhat-Szary, Didier Paris, Bernard Reitel et Olivier Walther

\section{(2) OpenEdition}

Journals

Édition électronique

URL : http://journals.openedition.org/belgeo/10659

DOI : 10.4000/belgeo.10659

ISSN : 2294-9135

Éditeur :

National Committee of Geography of Belgium, Société Royale Belge de Géographie

\section{Référence électronique}

Grégory Hamez, Anne-Laure Amilhat-Szary, Didier Paris, Bernard Reitel et Olivier Walther, « Guest editorial : Modèles de frontières », Belgeo [En ligne], 1 | 2013, mis en ligne le 31 octobre 2013, consulté le 22 septembre 2020. URL : http://journals.openedition.org/belgeo/10659 ; DOI : https://doi.org/ 10.4000/belgeo. 10659

Ce document a été généré automatiquement le 22 septembre 2020.

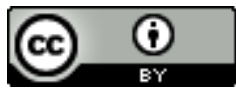

Belgeo est mis à disposition selon les termes de la licence Creative Commons Attribution 4.0 International. 


\title{
Guest editorial : Modèles de frontières
}

\author{
Grégory Hamez, Anne-Laure Amilhat-Szary, Didier Paris, Bernard Reitel et \\ Olivier Walther
}

1 L'étude des espaces transfrontaliers se fait souvent en référence à un contexte propre, dans la mesure où chaque frontière est le fruit d'une histoire particulière, des processus de construction des Etats en présence et de leurs relations. Ce poids important du contexte nourrit des controverses scientifiques sur la possibilité même d'élaborer une théorie générale des frontières. Si plusieurs auteurs ont contribué à une telle théorie au cours des dernières décennies (parmi lesquels Raffestin, 1986; Ratti et Reichmann, 1993 ; Brunet-Jailly, 2005), d'autres considèrent qu'un tel exercice théorique n'aurait de sens qu'au sein d'une théorie globale des processus de territorialisation (Newman, 2006 ; Paasi, 2005). Le numéro spécial de Belgeo s'inscrit dans ce débat, en questionnant la possibilité d'élaborer des modèles de frontières et d'espaces transfrontaliers.

2 L'expression modèle de frontières recouvre en première approche le sens d'une représentation menée à des fins scientifiques. En partant de la définition proposée par Peter Haggett, est considéré comme modèle : "a simplified version of reality, built in order to demonstrate certain of the properties of reality" (Haggett, 1965). La modélisation suppose donc une simplification dans le sens de distinguer les processus fondamentaux des processus secondaires; elle s'inscrit dans une démarche de démonstration et de production d'hypothèses propre à la recherche scientifique; elle suppose enfin un effort de clarté dans les propos, dans la mesure où le modèle doit rester intelligible (Brunet, 2001). L'approche modélisatrice implique souvent un volet quantitatif, difficile à mettre en œuvre dans les situations frontalières où les critères ne sont pas produits de façon homogène, et où les questions sensibles de représentation et de perception jouent un rôle important. La question concerne donc aussi la place à accorder aux démarches qualitatives dans ces modèles, et éventuellement à la possibilité de mixer les deux types de démarche.

3 Elaborer des modèles des frontières est un exercice périlleux au regard de la complexité de l'objet et de ses changements. De fait, les frontières évoluent dans leurs 
formes spatiales et dans leurs fonctions : à la ligne fixe se substitue un réseau de contrôle construit sur tous les points d'entrée du territoire national, permettant un suivi des mouvements et itinéraires (Retaillé, 2011); à la fonction de surveillance se substitue une fonction de contrôle, de plus en plus ubiquiste et permanent (Amoore, 2011). Les préoccupations des Etats en matière de contrôle du mouvement entrent en contradiction avec l'accroissement quantitatif des flux de marchandises qui résultent de la mondialisation (flux tendus, délocalisations, relocalisations, global chains), et des flux humains qui accompagnent la croissance des grandes régions métropolitaines et leurs exigences combinées de main-d'œuvre très qualifiée et peu qualifiée. Il en résulte un paradoxe entre ouverture et contrôle: "Borders now have to allow mobility while simultaneously protecting against its side effects" (Popescu, 2012).

4 Dans cette évolution, les effets des frontières se complexifient et se manifestent dans les différents domaines de la vie des individus. Les chercheurs participant à leur étude appartiennent à des disciplines scientifiques variées, notamment l'économie, la géographie, les sciences politiques et la sociologie en ce qui concerne les auteurs participant à ce numéro. L'interdisciplinarité est d'ailleurs un des enjeux du champ de recherche en émergence des border studies (Wilson et Donnan, 2012). A cet égard, la production de modèles n'est pas aisée en termes de dialogue entre des conceptions différentes de la frontière. Si dans certaines disciplines comme la géographie ou l'économie, la frontière nationale est considérée comme un objet d'étude, il s'agit davantage d'une catégorie analytique dans des disciplines comme la sociologie ou l'anthropologie (Jeanpierre, 2012). Dans le premier cas, l'intérêt se porte sur la force de cloisonnement et de discontinuité des frontières nationales, leur rôle pour freiner ou accélérer les flux, voire la façon dont les populations produisent et reproduisent les frontières à travers leurs actions et pratiques. Dans le second cas, le terme de "frontière" recouvre toute forme de contact, d'échange, de conflit entre des groupes et leurs cultures, identités et symboles - en cherchant comment les groupes sociaux construisent leurs limites, et les utilisent pour réguler leurs interactions. La modélisation commence par le langage ; pour progresser vers un modèle et permettre le dialogue interdisciplinaire, les auteurs réunis ici apportent différents lexiques illustrant la façon dont ils envisagent les frontières.

Les douze articles constituant ce numéro spécial de Belgeo illustrent plusieurs façons de modéliser les frontières. Dans les trois premiers, les auteurs adoptent une approche déductive, partant de constructions conceptuelles relatives aux principales caractéristiques des frontières et des processus qui s'y jouent, pour déboucher sur des typologies de frontières - tout en suggérant quelques démarches méthodologiques et nouvelles pistes de recherche ouvertes par le modèle. Dans les articles suivants, la plupart des auteurs privilégient une approche inductive en partant de processus constatés sur le terrain, ou bien d'instruments de gouvernance, pour en inférer des régularités représentées sous forme de graphiques, de schémas ou encore de grilles de lecture comparative. Plus rares sont les auteurs optant pour une approche hypothéticodéductive et un aller-retour entre propositions théoriques et application méthodologique - et leur rareté est probablement due à ce que l'accès à des données comparables et harmonisées s'avère toujours éminemment difficile en contexte transfrontalier. Le panorama offert par les douze auteurs est varié en termes d'échelle d'analyse, allant du niveau mondial pour certains articles, au cadre européen voire bilatéral entre deux pays pour d'autres. 
$6 \quad$ Les deux premiers articles proposent un essai de théorisation globale des frontières à l'échelle du monde. L'article de Vladimir Kolossov et James Scott, qui ouvre ce numéro, aborde la modélisation comme détermination de processus et d'invariants en cours sur les frontières, en se concentrant sur la dialectique entre processus de contrôle et de souveraineté étatique d'une part, et d'interrelations sociales dans les pratiques quotidiennes d'autre part. En situant leur propos au sein de la littérature abondante constituant les border studies, les auteurs soulignent l'importance d'une échelle intermédiaire - l'échelle des régions frontalières - pour cerner la relation entre processus étatiques et sociaux, et énoncent plusieurs problématiques essentielles telles que le jeu entre atténuation et renforcement des frontières (debordering/rebordering), le croisement entre haute et basse géopolitique ou la difficile conciliation entre les impératifs sécuritaires et éthiques. Le deuxième article, de Remigio Ratti et Martin Schuler, propose un modèle basé sur les processus apparus au cours des dernières décennies qui ont conduit à engendrer la variété des types de frontières dans le monde. La thèse centrale de l'article est qu'il est impossible de concevoir les frontières comme des objets statiques et immuables. En croisant trois formes spatiales des frontières (ligne, zone, lieux globaux) avec trois types de dynamiques relatives à leurs effets (fixité, mobilité, ouverture sur l'horizon), les deux auteurs produisent un tableau où chacun des neuf quadrants correspond à un type particulier de frontière.

7 La question des frontières est abordée dans le cadre européen pour les trois articles suivants, en s'appliquant aux espaces transfrontaliers. Compte tenu de l'incohérence sémantique inhérente au terme de territoire transfrontalier, Grégory Hamez centre son propos sur les territoires frontaliers; ces derniers sont analysés à travers un modèle croisant trois échelles spatiales (local, national, européen) et trois composantes du territoire (pratiques spatiales, gouvernance et représentations). La spatialisation du modèle ouvre plusieurs pistes de recherche, telles que le lien éventuel entre pratiques locales transfrontalières et horizon européen de représentation, ou entre pratiques transfrontalières et gouvernance européenne. De fait, la gouvernance dans les espaces transfrontaliers est également un riche champ d'investigations : Valérie Biot étudie les groupements européens de coopération territoriale (GECT) de façon comparative, et Estelle Evrard interroge les conditions nécessaires à la mise en place d'une région réunissant les deux côtés de la frontière. Valérie Biot propose une grille de lecture du GECT, qui ouvre sur une vision globale de ce nouvel instrument européen de coopération, tandis qu'Estelle Evrard évoque deux processus clefs pour la construction transfrontalière d'une région, l'institutionnalisation et la territorialisation (entendue comme "appropriation").

8 La modélisation peut également passer par des études monographiques, à condition que les études de cas permettent de poser les bases d'une généralisation. L'article de Sarolta Németh, Ágnes Németh et Virpi Kaisto, souligne les enjeux et les limites de l'analyse comparative à partir de l'exemple de quatre projets de recherche européens ayant trait aux frontières, les projets ESPON Ulysses et Terco, le projet FP7 Euborderregions et le projet ESF Unfamiliarity. Il est aussi possible d'inférer des constantes à partir d'un seul cas, comme le fait Adenane Djigo dans son article consacré à la partie orientale de la frontière transpyrénéenne, où il montre le rôle fondamental de la proximité pour légitimer la rhétorique de la coopération transfrontalière.

Des contributions vers une modélisation qualitative sont données dans les deux articles suivants. Heidi Fichter-Wolff soulève la question du transfert culturel dans les espaces 
transfrontaliers, à travers les interactions et les communications entre individus porteurs de cultures différentes. En appliquant l'interrogation dans le cas des relations entre établissements d'enseignement supérieur (entre Allemagne et Danemark, entre Allemagne et Pologne), l'analyse laisse entrevoir les possibilités "d'apprentissage organisationnel". François Moullé adopte une démarche située au croisement de la psychologie de l'espace et de la géographie, pour mettre en évidence le double mouvement de fluidification des frontières et de renforcement local d'enclaves (gated communities et privatisation de l'espace public). A la frontière extérieure de l'espace Schengen, apportant une protection lointaine, répond la frontière locale des enclaves, apportant une protection de proximité.

10 Les trois derniers articles constituent une ouverture sur les modélisations possibles d'autres types de frontières. A partir d'une vaste enquête portant sur la façon dont les étudiants européens et extra-européens perçoivent les limites de l'Europe (PCRD Eurobroadmap), Sophie de Ruffray applique la méthodologie des cartes mentales interprétatives pour mettre en évidence la variabilité de ces limites en fonction de la nationalité des enquêtés, et de leur situation à l'intérieur ou à l'extérieur de ce qui est considéré comme "Europe". Autre type de limite, celui du détroit, en l'occurrence le détroit de Gibraltar étudié par Nora Mareï et Nacima Baron-Yelles. Les auteurs proposent trois modèles de fonctionnement pour illustrer les différentes formes de cette figure de l'entre-deux. Enfin, Antoine Laporte aborde une frontière relique, l'ancien Mur de Berlin : bien que disparue, sa trace demeure prégnante et se retrouve dans l'architecture des bâtiments nouvellement construits, ainsi que dans les pratiques spatiales.

11 Il est un autre sens du mot modèle, peu abordé dans les articles rassemblés ici : le sens d'exemplarité. La question serait de savoir si certaines frontières peuvent servir d'exemple, ou si certains cas de développement transfrontalier pourraient être transposés ailleurs. Le modèle peut circuler, tout en s'hybridant (Amilhat-Szary et Koop, 2011). La question est d'importance pour les frontières: en effet, la représentation dominante de la frontière est celle du monde occidental, parfois appelée westphalienne au regard du contexte historique de son émergence. Les formes des frontières dans les autres parties du monde peuvent sensiblement s'éloigner de ce modèle dominant, mais la recherche scientifique actuelle a-t-elle les moyens de l'observer? La grande majorité des études sur les frontières porte sur les frontières du monde occidental (figure 1); et les chercheurs qui mènent ces études sont majoritairement des Occidentaux, dont les conceptions sont liées aux sociétés dans lesquelles ils vivent. Il y a un enjeu majeur au décentrement épistémologique et culturel. 
Figure 1. Pays dont les frontières sont les plus étudiées, selon la base bibliographique EBSCO.

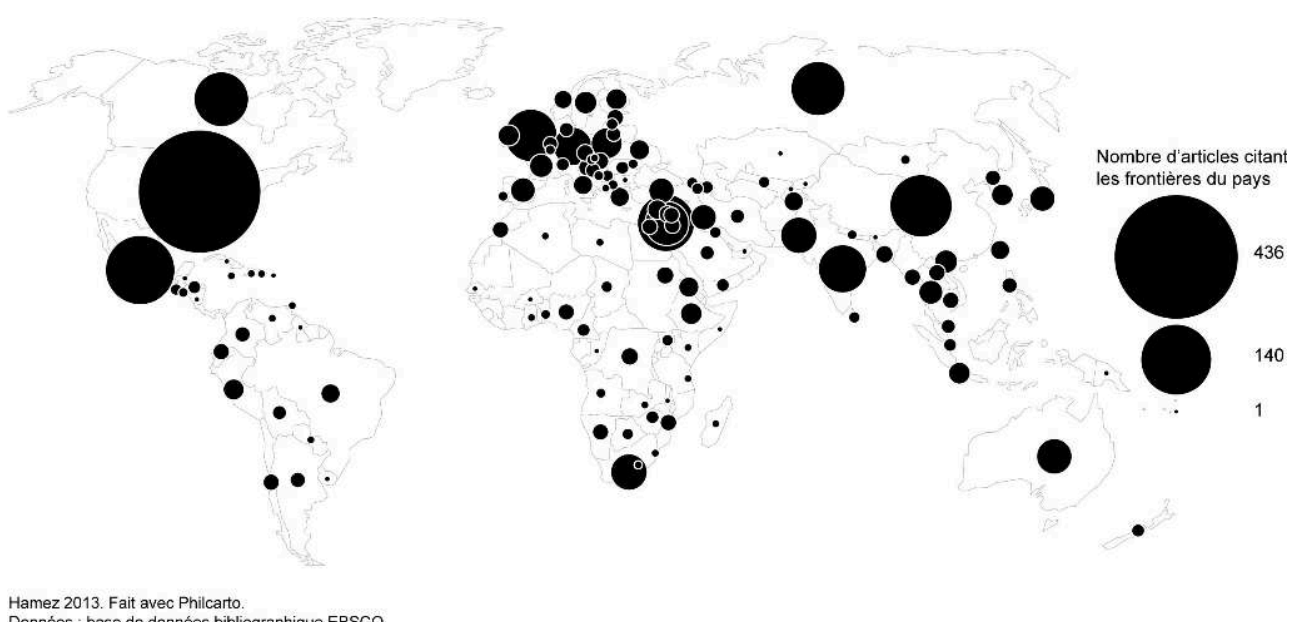

Hamez 2013. Fait avec Philcarto.

Note : La base EBSCO a été analysée systématiquement pour les années 2000 à 2010. Ont été considérés tous les articles scientifiques ayant comme mot-clef "boundary" ou "border", au sens de limite territoriale (2573 au total). Quand la frontière analysée est la limite entre plusieurs pays, chacun des pays compte pour un article. La base EBSCO comprenant principalement des ouvrages en anglais, cette carte livre une représentation de la recherche anglo-saxonne sur les frontières.

\section{BIBLIOGRAPHIE}

AMILHAT-SZARY A.-L., KOOP K. (2011), “Introduction. Approche critique des transferts contemporains des modèles de développement territorial vers les Suds", L'information géographique, 75, 4, pp. 6-14.

AMOORE L. (2009), “Algorithmic war: everyday geographies of the war on terror", Antipode: A Radical Journal of Geography, 41, pp. 49-69.

BRUNET R. (2001), "Models in geography? A sense to research", Cybergeo : European Journal of Geography [En ligne], document 204, mis en ligne le 18 décembre 2001, consulté le 17 octobre 2013, http://cybergeo.revues.org/4288.

BRUNET-JAILLY E. (2005), “Theorizing Borders: An Interdisciplinary Perspective”, Geopolitics, 10, pp. 633-649.

HAGGETT P. (1965), Locational Analysis in Human Geography, Londres, E. Arnold.

JEANPIERRE L. (2010), “Frontière”, in CHRISTIN O. (dir.), Dictionnaire des concepts nomades en sciences humaines, Métailié, Paris, pp. 157-169.

PAASI A. (2005), “Generations and the 'Development' of Border Studies”, Geopolitics, 10, pp. 663-671.

POPESCU G. (2012), Bordering and Ordering in the Twenty-first Century: Understanding borders, Plymouth, Rowman \& Littlefield. 
RAFFESTIN C. (1986), "Eléments pour une théorie de la frontière”, Diogène, 34, 134, pp. 3-21, disponible sur http://archive-ouverte.unige.ch/unige:4348, consulté le 31 octobre 2013.

RATTI R., REICHMANN S. (1993), Theory and strategy of border areas development, Bâle et Francfort s/Main, Helbling \& Lichtenbahn.

RETAILLE D. (2011), "La transformation des formes de la limite", Articulo - Journal of Urban Research, http://articulo.revues.org/1723, DOI : 10.400/articulo.1723, consulté le 31 octobre 2013.

WILSON Th. M., DONNAN H. (2012), “Borders and border studies”, in WILSON Th. M., Donnan H. (eds.), A companion to border studies, Londres, Wiley-Blackwell. 